\title{
A Shrinking Projection Method for Generalized Mixed Equilibrium Problems, Variational Inclusion Problems and a Finite Family of Quasi-Nonexpansive Mappings
}

\author{
Wiyada Kumam, ${ }^{1,2}$ Chaichana Jaiboon, ${ }^{3}$ Poom Kumam, ${ }^{2,4}$ \\ and Akarate Singta ${ }^{1}$ \\ ${ }^{1}$ Department of Mathematics and Computer Science, Faculty of Science and Technology, \\ Rajamangala University of Technology Thanyaburi (RMUTT), Thanyaburi, Pathumthani 12110, Thailand \\ ${ }^{2}$ Centre of Excellence in Mathematics CHE, Si Ayutthaya Road, Bangkok 10140, Thailand \\ ${ }^{3}$ Department of Mathematics, Faculty of Liberal Arts, Rajamangala University of Technology Rattanakosin \\ (RMUTR), Bangkok 10100, Thailand \\ ${ }^{4}$ Department of Mathematics, Faculty of Science, King Mongkut's University of Technology Thonburi \\ (KMUTT), Bangkok 10140, Thailand
}

Correspondence should be addressed to Chaichana Jaiboon, chaichana.j@rmutr.ac.th

Received 21 March 2010; Accepted 29 June 2010

Academic Editor: Jong Kim

Copyright (c) 2010 Wiyada Kumam et al. This is an open access article distributed under the Creative Commons Attribution License, which permits unrestricted use, distribution, and reproduction in any medium, provided the original work is properly cited.

The purpose of this paper is to consider a shrinking projection method for finding a common element of the set of solutions of generalized mixed equilibrium problems, the set of fixed points of a finite family of quasi-nonexpansive mappings, and the set of solutions of variational inclusion problems. Then, we prove a strong convergence theorem of the iterative sequence generated by the shrinking projection method under some suitable conditions in a real Hilbert space. Our results improve and extend recent results announced by Peng et al. (2008), Takahashi et al. (2008), S.Takahashi and W. Takahashi (2008), and many others.

\section{Introduction}

Throughout this paper, we assume that $H$ is a real Hilbert space with inner product $\langle\cdot, \cdot\rangle$ and norm $\|\cdot\|$, and let $C$ be a nonempty closed convex subset of $H$. We denote weak convergence and strong convergence by notations $\rightarrow$ and $\rightarrow$, respectively.

Recall that the following definitions.

(1) A mapping $T: C \rightarrow C$ is said to be nonexpansive if

$$
\|T x-T y\| \leq\|x-y\|, \quad \forall x, y \in C .
$$


(2) A mapping $T: C \rightarrow C$ is said to be quasi-nonexpansive if

$$
\|T x-p\| \leq\|x-p\|, \quad \forall x \in C, p \in F(T) .
$$

We denote $F(T)=\{x \in C: T x=x\}$ be the set of fixed points of $T$.

Let $B: H \rightarrow H$ be a single-valued nonlinear mapping and $M: H \rightarrow 2^{H}$ to a setvalued mapping. The variational inclusion problem is to find $\widehat{x} \in H$ such that

$$
\theta \in B(\widehat{x})+M(\widehat{x})
$$

where $\theta$ is the zero vector in $H$. The set of solutions of problem (1.3) is denoted by $I(B, M)$.

Definition 1.1. A mapping $B: H \rightarrow H$ is said to be a $\xi$-inverse-strongly monotone if there exists a constant $\xi>0$ with the property

$$
\langle B x-B y, x-y\rangle \geq \xi\|B x-B y\|^{2}, \quad \forall x, y \in C .
$$

Remark 1.2. It is obvious that any $\xi$-inverse-strongly monotone mapping $B$ is monotone and $1 / \xi$-Lipschitz continuous. It is easy to see that if any $\lambda$ constant is in $(0,2 \xi]$, then the mapping $I-\lambda B$ is nonexpansive, where $I$ is the identity mapping on $H$.

A set-valued mapping $M: H \rightarrow 2^{H}$ is called monotone if for all $x, y \in H, f \in M(x)$, and $g \in M(y)$ implying $\langle x-y, f-g\rangle \geq 0$. A monotone mapping; $M$ is maximal if its graph $G(M):=\{(f, x) \in H \times H: f \in M(x)\}$ of $M$ is not properly contained in the graph of any other monotone mapping. It is known that a monotone mapping $M$ is maximal if and only if for $(x, f) \in H \times H,\langle x-y, f-g\rangle \geq 0$ for all $(y, g) \in G(M)$ imply $f \in M(x)$.

Definition 1.3. Let $M: H \rightarrow 2^{H}$ be a set-valued maximal monotone mapping, then the singlevalued mapping $J_{M, \lambda}: H \rightarrow H$ defined by

$$
J_{M, \lambda}(\widehat{x})=(I+\lambda M)^{-1}(\widehat{x}), \quad \widehat{x} \in H
$$

is called the resolvent operator associated with $M$, where $\lambda$ is any positive number and $I$ is the identity mapping.

Remark 1.4. (R1) The resolvent operator $J_{M, \lambda}$ is single-valued and nonexpansive for all $\lambda>0$, that is,

$$
\left\|J_{M, \lambda}(x)-J_{M, \lambda}(y)\right\| \leq\|x-y\|, \quad \forall x, y \in H, \forall \lambda>0 .
$$

(R2) The resolvent operator $J_{M, \lambda}$ is 1-inverse strongly monotone; see [1], that is,

$$
\left\|J_{M, \lambda}(x)-J_{M, \lambda}(y)\right\|^{2} \leq\left\langle J_{M, \lambda}(x)-J_{M, \lambda}(y), x-y\right\rangle, \quad \forall x, y \in H
$$


(R3) The solution of problem (1.3) is a fixed point of the operator $J_{M, \lambda}(I-\lambda B)$ for all $\lambda>0$; see also [2], that is,

$$
I(B, M)=F\left(J_{M, \lambda}(I-\lambda B)\right), \quad \forall \lambda>0 .
$$

(R4) If $0<\lambda \leq 2 \xi$, then the mapping $J_{M, \lambda}(I-\lambda B): H \rightarrow H$ is nonexpansive.

(R5) $I(B, M)$ is closed and convex.

Let $A: C \rightarrow H$ be a nonlinear mapping, let $\varphi: C \rightarrow \mathbb{R} \cup\{+\infty\}$ be a real-valued function and $F$ a bifunction from $C \times C$ to $\mathbb{R}$. We consider the following generalized mixed equilibrium problem.

Finding $x \in C$ such that

$$
F(x, y)+\langle A x, y-x\rangle+\varphi(y)-\varphi(x) \geq 0, \quad \forall y \in C
$$

The set of such $x \in C$ is denoted by $\operatorname{GMEP}(F, \varphi, A)$, that is,

$$
\operatorname{GMEP}(F, \varphi, A)=\{x \in C: F(x, y)+\langle A x, y-x\rangle+\varphi(y)-\varphi(x) \geq 0, \forall y \in C\}
$$

It is easy to see that $x$ is solution of problem (1.9) implies that $x \in \operatorname{dom} \varphi=\{x \in C: \varphi(x)<$ $+\infty\}$.

(i) In the case of $A \equiv 0$ (:the zero mapping), then the generalized mixed equilibrium problem (1.9) is reduced to the mixed equilibrium problem. Finding $x \in C$ such that

$$
F(x, y)+\varphi(y)-\varphi(x) \geq 0, \quad \forall y \in C
$$

The set of solution of (1.11) is denoted by $\operatorname{MEP}(F, \varphi)$.

(ii) In the case of $\varphi \equiv 0$, then the generalized mixed equilibrium problem (1.9) is reduced to the generalized equilibrium problem. Finding $x \in C$ such that

$$
F(x, y)+\langle A x, y-x\rangle \geq 0, \quad \forall y \in C
$$

The set of solution of (1.12) is denoted by $\operatorname{GEP}(F, A)$.

(iii) In the case of $A \equiv 0$ (:the zero mapping) and $\varphi \equiv 0$, then the generalized mixed equilibrium problem (1.9) is reduced to the equilibrium problem. Finding $x \in C$ such that

$$
F(x, y) \geq 0, \quad \forall y \in C
$$

The set of solution of (1.13) is denoted by $\operatorname{EP}(F)$. 
(iv) In the case of $F \equiv 0, \varphi \equiv 0$ and $A \equiv B$ then the generalized mixed equilibrium problem (1.9) is reduced to the variational inequality problem. Finding $x \in C$ such that

$$
\langle B x, y-x\rangle \geq 0, \quad \forall y \in C
$$

The set of solution of (1.14) is denoted by $\mathrm{VI}(C, B)$.

The generalized mixed equilibrium problem include fixed point problems, optimization problems, variational inequalities problems, Nash equilibrium problems, noncooperative games, economics and the equilibrium problems as special cases (see, e.g., [3-8]). Some methods have been proposed to solve the generalized mixed equilibrium problems, generalized equilibrium problems and equilibrium problems; see, for instance, [9-22].

In 2007, Takahashi et al. [23] proved the following strong convergence theorem for a nonexpansive mapping by using the shrinking projection method in mathematical programming. For a $C_{1}=C$ and $x_{1}=P_{C_{1}} x_{0}$, they defined a sequence as follows

$$
\begin{gathered}
y_{n}=\alpha_{n} x_{n}+\left(1-\alpha_{n}\right) T x_{n}, \\
C_{n+1}=\left\{z \in C_{n}:\left\|y_{n}-z\right\| \leq\left\|x_{n}-z\right\|\right\}, \\
x_{n+1}=P_{C_{n+1}} x_{0}, \quad \forall n \geq 1,
\end{gathered}
$$

where $0 \leq \alpha_{n}<a<1$. They proved that the sequence $\left\{x_{n}\right\}$ generated by (1.15) converges weakly to $z \in F(T)$, where $z=P_{F(T)} x_{0}$.

In 2008, S. Takahashi and W. Takahashi [24] introduced the following iterative scheme for finding a common element of the set of solutions of mixed equilibrium problems and the set of fixed points of a nonexpansive mapping in a Hilbert space. Starting with arbitrary $x_{1}=u \in C$, define sequences $\left\{x_{n}\right\},\left\{y_{n}\right\}$ and $\left\{u_{n}\right\}$ by

$$
\begin{gathered}
F\left(u_{n}, y\right)+\left\langle A x_{n}, y-u_{n}\right\rangle+\frac{1}{r_{n}}\left\langle y-u, u_{n}-x_{n}\right\rangle \geq 0, \quad \forall y \in C \\
y_{n}=\alpha_{n} u+\left(1-\alpha_{n}\right) u_{n} \\
x_{n+1}=\beta_{n} x_{n}+\left(1-\beta_{n}\right) T y_{n} .
\end{gathered}
$$

They proved that under certain appropriate conditions imposed on $\left\{\alpha_{n}\right\},\left\{\beta_{n}\right\}$ and $\left\{r_{n}\right\}$, the sequence $\left\{x_{n}\right\}$ generated by (1.16) converges strongly to $z=P_{F(T) \cap \operatorname{GEP}(F, A)} u$.

In 2008, Zhang et al. [25] introduced the following new iterative scheme for finding a common element of the set of solutions to the problem (1.3) and the set of fixed points of a nonexpansive mapping in a real Hilbert space. Starting with an arbitrary $x_{1}=x \in H$, define sequences $\left\{x_{n}\right\}$ and $\left\{y_{n}\right\}$ by

$$
\begin{gathered}
y_{n}=J_{M, \lambda}\left(x_{n}-\lambda B x_{n}\right), \\
x_{n+1}=\alpha_{n} x+\left(1-\alpha_{n}\right) T y_{n}, \quad \forall n \geq 0,
\end{gathered}
$$

where $J_{M, \lambda}=(I+\lambda M)^{-1}$ is the resolvent operator associated with $M$ and a positive number $\lambda,\left\{\alpha_{n}\right\}$ is a sequence in the interval $[0,1]$. 
In 2008, Peng et al. [26] introduced the following iterative scheme by the viscosity approximation method for finding a common element of the set of solutions to the problem (1.3), the set of solutions of an equilibrium problems and the set of fixed points of nonexpansive mappings in a Hilbert space. Starting with an arbitrary $x_{1} \in H$, define sequences $\left\{x_{n}\right\},\left\{y_{n}\right\}$ and $\left\{u_{n}\right\}$ by

$$
\begin{gathered}
F\left(u_{n}, y\right)+\frac{1}{r_{n}}\left\langle y-u_{n}, u_{n}-x_{n}\right\rangle \geq 0, \quad \forall y \in C, \\
y_{n}=J_{M, \lambda}\left(u_{n}-\lambda B u_{n}\right), \\
x_{n+1}=\alpha_{n} f\left(x_{n}\right)+\left(1-\alpha_{n}\right) T y_{n}, \quad \forall n \geq 0 .
\end{gathered}
$$

They proved that under certain appropriate conditions imposed on $\left\{\alpha_{n}\right\}$ and $\left\{r_{n}\right\}$, the sequence $\left\{x_{n}\right\}$ generated by (1.18) converges strongly to $z=P_{F(T) \cap \operatorname{EP}(F) \cap I(B, M)} f(z)$.

In 2010, Katchang and Kumam [27] introduced an iterative scheme for finding a common element of the set of solutions for mixed equilibrium problems, the set of solutions of the variational inclusions with set-valued maximal monotone mappings, and inverse strongly monotone mappings and the set of fixed points of a finite family of nonexpansive mappings in a real Hilbert space.

In this paper, motivated and inspired by the previously mentioned results, we introduce an iterative scheme by the shrinking projection method for finding a common element of the set of solutions of generalized mixed equilibrium problems, the set of fixed points of a finite family of quasi-nonexpansive mappings and the set of solutions of variational inclusion problems in a real Hilbert space. Then, we prove a strong convergence theorem of the iterative sequence generated by the proposed shrinking projection method under some suitable conditions. The results obtained in this paper extend and improve several recent results in this area.

\section{Preliminaries}

Let $H$ be a real Hilbert space and let $C$ be a nonempty closed convex subset of $H$. Recall that the (nearest point) projection $P_{C}$ from $H$ onto $C$ assigns to each $x \in H$ the unique point in $P_{C} x \in C$ satisfying the property

$$
\left\|x-P_{C} x\right\|=\min _{y \in C}\|x-y\| .
$$

We recall some lemmas which will be needed in the rest of this paper.

Lemma 2.1. For a given $x \in H$ and $z \in C$,

$$
z=P_{C} x \Longleftrightarrow\langle x-z, z-y\rangle \geq 0, \quad \forall y \in C
$$

It is well known that $P_{C}$ is a firmly nonexpansive mapping of $H$ onto $C$ and satisfies

$$
\left\|P_{C} x-P_{C} y\right\|^{2} \leq\left\langle P_{C} x-P_{C} y, x-y\right\rangle, \quad \forall x, y \in H .
$$


Lemma 2.2 (see [1]). Let $M: H \rightarrow 2^{H}$ be a maximal monotone mapping and let $B: H \rightarrow H$ be a Lipshitz continuous mapping. Then the mapping $S=M+B: H \rightarrow 2^{H}$ is a maximal monotone mapping.

Lemma 2.3 (see [28]). Let $C$ be a closed convex subset of $H$ and let $\left\{x_{n}\right\}$ be a bounded sequence in H. Assume that

(1) the weak $\omega$-limit set $\omega_{w}\left(x_{n}\right) \subset C$,

(2) for each $z \in C, \lim _{n \rightarrow \infty}\left\|x_{n}-z\right\|$ exists.

Then $\left\{x_{n}\right\}$ is weakly convergent to a point in $C$.

Lemma 2.4 (see [29]). Each Hilbert space $H$ satisfies Opial's condition, that is, for any sequence $\left\{x_{n}\right\} \subset H$ with $x_{n} \rightarrow x$, the inequality

$$
\liminf _{n \rightarrow \infty}\left\|x_{n}-x\right\|<\liminf _{n \rightarrow \infty}\left\|x_{n}-y\right\|
$$

holds for each $y \in H$ with $y \neq x$.

Lemma 2.5 (see [30]). Each Hilbert space $H$ satisfies the Kadec-Klee property, that is, for any sequence $\left\{x_{n}\right\}$ with $x_{n} \rightarrow x$ and $\left\|x_{n}\right\| \rightarrow\|x\|$ together imply $\left\|x_{n}-x\right\| \rightarrow 0$.

For solving the generalized equilibrium problems, let us give the following assumptions for $F, \varphi$, and the set $C$ :

(A1) $F(x, x)=0$ for all $x \in C$;

(A2) $F$ is monotone, that is, $F(x, y)+F(y, x) \leq 0$ for all $x, y \in C$;

(A3) for each $y \in C, x \mapsto F(x, y)$ is weakly upper semicontinuous;

(A4) for each $x \in C, y \mapsto F(x, y)$ is convex and lower semicontinuous;

(B1) for each $x \in H$ and $r>0$, there exists a bounded subset $D_{x} \subseteq C$ and $y_{x} \in C \cap \operatorname{dom} \varphi$ such that for any $z \in C \backslash D_{x}$,

$$
F\left(z, y_{x}\right)+\varphi\left(y_{x}\right)-\varphi(z)+\frac{1}{r}\left\langle y_{x}-z, z-x\right\rangle \geq 0
$$

(B2) C is bounded set.

Lemma 2.6 (see [31]). Let $C$ be a nonempty closed convex subset of $H$ and let $F$ be a bifunction of $C \times C$ into $\mathbb{R}$ satisfying (A1)-(A4). Let $\varphi: H \rightarrow R \cup\{+\infty\}$ be a proper lower semicontinuous and convex function such that $C \cap \operatorname{dom} \neq \emptyset$. For $r>0$ and $x \in H$, define a mapping $T_{r}: H \rightarrow C$ as follows:

$$
T_{r}(x)=\left\{z \in C: F(z, y)+\varphi(y)-\varphi(z)+\frac{1}{r}\langle y-z, z-x\rangle \geq 0, \forall y \in C\right\} .
$$


Assume that either (B1) or (B2) holds. Then, the following conclusions hold:

(1) for each $x \in H, \quad T_{r}(x) \neq \emptyset$;

(2) $T_{r}$ is single-valued;

(3) $T_{r}$ is firmly nonexpansive, that is, for any $x, y \in H$,

$$
\left\|T_{r}(x)-T_{r}(y)\right\|^{2} \leq\left\langle T_{r}(x)-T_{r}(y), x-y\right\rangle
$$

(4) $F\left(T_{r}\right)=G E P(F, \varphi)$;

(5) $\operatorname{GEP}(F, \varphi)$ is closed and convex.

Remark 2.7. Replacing $x$ with $x-r A x \in H$ in (2.5), then there exists $z \in C \backslash D_{x}$, such that

$$
F\left(z, y_{x}\right)+\left\langle A x, y_{x}-z\right\rangle+\varphi\left(y_{x}\right)-\varphi(z)+\frac{1}{r}\left\langle y_{x}-z, z-x\right\rangle \geq 0, \quad \forall y \in C
$$

\section{Main Results}

In this section, we will introduce an iterative scheme by using shrinking projection method for finding a common element of the set of solutions of generalized mixed equilibrium problems, the set of fixed points of a finite family of quasi-nonexpansive mappings and the set of solutions of variational inclusion problems in a real Hilbert space.

Let $\left\{T_{i}\right\}_{i=1}^{N}$ be a finite family of nonexpansive mappings of $C$ into itself, and let $\gamma_{1}, \ldots, \gamma_{N}$ be real numbers such that $0 \leq \gamma_{i} \leq 1$ for every $i=1, \ldots, N$. We define a mapping $K: C \rightarrow C$ as follows:

$$
\begin{aligned}
U_{1} & =\gamma_{1} T_{1}+\left(1-\gamma_{1}\right) I, \\
U_{2} & =\gamma_{2} T_{2} U_{1}+\left(1-\gamma_{2}\right) I, \\
U_{3} & =\gamma_{3} T_{3} U_{2}+\left(1-\gamma_{3}\right) I, \\
& \vdots \\
U_{N-1} & =\gamma_{N-1} T_{N-1} U_{N-2}+\left(1-\gamma_{N-1}\right) I, \\
K & =U_{N}=\gamma_{N} T_{N} U_{N-1}+\left(1-\gamma_{N}\right) I .
\end{aligned}
$$

Such a mapping $K$ is called the $K$-mapping generated by $T_{1}, \ldots, T_{N}$ and $\gamma_{1}, \ldots, \gamma_{N}$; see [32].

We have the following crucial Lemma 3.1 and Lemma 3.2 concerning $K$-mapping which can be found in [14]. Now we only need the following similar version in Hilbert spaces.

Lemma 3.1. Let $C$ be a nonempty closed convex subset of a real Hilbert space $H$. Let $\left\{T_{i}\right\}_{i=1}^{N}$ be a finite family of quasi-nonexpansive mappings and $L_{i}$-Lipschitz mappings of $C$ into itself with $\bigcap_{i=1}^{N} F\left(T_{i}\right) \neq \emptyset$ and let $\gamma_{1}, \ldots, \gamma_{N}$ be real numbers such that $0<\gamma_{i}<1$ for every $i=1, \ldots, N-1,0<\gamma_{N} \leq 1$ and 
$\sum_{i=1}^{N}=1$. Let $K$ be the K-mapping generated by $T_{1}, \ldots, T_{N}$ and $\gamma_{1}, \ldots, \gamma_{N}$. Then, the followings hold:

(1) $K$ is quasi-nonexpansive and Lipschitz,

(2) $F(K)=\bigcap_{i=1}^{N} F\left(T_{i}\right)$.

Lemma 3.2. Let $C$ be a nonempty closed convex subset of a real Hilbert space $H$. Let $\left\{T_{i}\right\}_{i=1}^{N}$ be a finite family of quasi-nonexpansive mappings and $L_{i}$-Lipschitz mappings of $C$ into itself and $\left\{\gamma_{n, i}\right\}_{i=1}^{N}$ sequences in $[0,1]$ such that $\gamma_{n, i} \rightarrow \gamma_{i}$, as $n \rightarrow \infty,(i=1,2, \ldots, N)$. Moreover, for every $n \in \mathbb{N}$, let $K$ and $K_{n}$ be the K-mappings generated by $T_{1}, T_{2}, \ldots, T_{N}$ and $\gamma_{1}, \gamma_{2}, \ldots, \gamma_{N}$, and $T_{1}, T_{2}, \ldots, T_{N}$ and $\gamma_{n, 1}, \gamma_{n, 2}, \ldots, \gamma_{n, N}$, respectively. Then, for every $x \in C$, we have $\lim _{n \rightarrow \infty}\left\|K_{n} x-K x\right\|=0$. method.

Now we study the strong convergence theorem concerning the shrinking projection

Theorem 3.3. Let $C$ be a nonempty closed convex subset of a real Hilbert space $H$, let $F$ be a bifunction from $C \times C$ to $\mathbb{R}$ satisfying (A1)-(A4), and let $\varphi: C \rightarrow \mathbb{R} \cup\{+\infty\}$ be a proper lower semicontinuous and convex function with assumption (B1) or (B2). Let $\left\{T_{i}\right\}_{i=1}^{N}$ be a finite family of quasi-nonexpansive and $L_{i}$-Lipschitz mappings of $C$ into itself, and let $A$ be a $\beta$-inverse-strongly monotone mapping of $C$ into $H$, let $B$ a $\xi$-inverse-strongly monotone mapping of $C$ into $H$ and $M: H \rightarrow 2^{H}$ be a maximal monotone mapping. Assume that

$$
\Theta:=\bigcap_{i=1}^{N} F\left(T_{i}\right) \cap \operatorname{GMEP}(F, \varphi, A) \cap I(B, M) \neq \emptyset .
$$

Let $K_{n}$ be the K-mapping generated by $T_{1}, T_{2}, \ldots, T_{N}$ and $\gamma_{n, 1}, \gamma_{n, 2}, \ldots, \gamma_{n, N}$. Let $\left\{x_{n}\right\},\left\{y_{n}\right\},\left\{v_{n}\right\}$, $\left\{z_{n}\right\}$ and $\left\{u_{n}\right\}$ be sequences generated by $x_{0} \in H, C_{1}=C, x_{1}=P_{C_{1}} x_{0}, u_{n} \in C$ and let

$$
\begin{gathered}
F\left(u_{n}, y\right)+\varphi(y)-\varphi\left(u_{n}\right)+\left\langle A x_{n}, y-u_{n}\right\rangle+\frac{1}{r_{n}}\left\langle y-u_{n}, u_{n}-x_{n}\right\rangle \geq 0, \quad \forall y \in C, \\
y_{n}=J_{M, \delta_{n}}\left(u_{n}-\delta_{n} B u_{n}\right), \\
v_{n}=J_{M, \lambda_{n}}\left(y_{n}-\lambda_{n} B y_{n}\right), \\
z_{n}=\alpha_{n} x_{n}+\left(1-\alpha_{n}\right) K_{n} v_{n}, \\
C_{n+1}=\left\{z \in C_{n}:\left\|z_{n}-z\right\| \leq\left\|x_{n}-z\right\|\right\}, \\
x_{n+1}=P_{C_{n+1}} x_{0}, \quad n \in \mathbb{N},
\end{gathered}
$$

where $\left\{\alpha_{n}\right\} \subset(0,1)$ satisfy the following conditions:

(i) $\left\{\alpha_{n}\right\} \subset[0, e]$ for some $e$ with $0 \leq e<1$;

(ii) $\left\{\delta_{n}\right\},\left\{\lambda_{n}\right\} \subset[a, b]$ for some $a, b$ with $0<a<b<2 \xi$;

(iii) $\left\{r_{n}\right\} \subset[c, d]$ for some $c, d$ with $0<c<d<2 \beta$.

Then, $\left\{x_{n}\right\}$ and $\left\{u_{n}\right\}$ converge strongly to $z=P_{\Theta} x_{0}$. 
Proof. In the light of the definition of the resolvent, $u_{n}$ can be rewritten as $u_{n}=T_{r_{n}}\left(x_{n}-\right.$ $\left.r_{n} A x_{n}\right)$. Let $p \in \Theta:=\cap_{i=1}^{N} F\left(T_{i}\right) \cap \operatorname{GMEP}(F, \varphi, A) \cap I(B, M)$, and using the fact $\left\{T_{r_{n}}\right\}$ be a sequence of mappings defined as in Lemma 2.6, $A$ is an $\beta$-inverse-strongly monotone and that $p=T_{\mathrm{r}_{n}}\left(p-r_{n} A p\right)$, where $\left\{r_{n}\right\} \subset[c, d]$ for some $c$, $d$ with $0<c<d<2 \beta$, we can write

$$
\begin{aligned}
\left\|u_{n}-p\right\|^{2} & =\left\|T_{r_{n}}\left(x_{n}-r_{n} A x_{n}\right)-T_{r_{n}}\left(p-r_{n} A p\right)\right\|^{2} \\
& \leq\left\|\left(x_{n}-r_{n} A x_{n}\right)-\left(p-r_{n} A p\right)\right\|^{2} \\
& =\left\|\left(x_{n}-p\right)-r_{n}\left(A x_{n}-A p\right)\right\|^{2} \\
& =\left\|x_{n}-p\right\|^{2}-2 r_{n}\left\langle x_{n}-p, A x_{n}-A p\right\rangle+r_{n}^{2}\left\|A x_{n}-A p\right\|^{2} \\
& \leq\left\|x_{n}-p\right\|^{2}-2 r_{n} \beta\left\|A x_{n}-A p\right\|^{2}+r_{n}^{2}\left\|A x_{n}-A p\right\|^{2} \\
& =\left\|x_{n}-p\right\|^{2}+r_{n}\left(r_{n}-2 \beta\right)\left\|A x_{n}-A p\right\|^{2} \\
& \leq\left\|x_{n}-p\right\|^{2} .
\end{aligned}
$$

Next, we will divide the proof into six steps.

Step 1. We first show that $\left\{x_{n}\right\}$ is well defined and $C_{n}$ is closed and convex for any $n \in \mathbb{N}$.

From the assumption, we see that $C_{1}=C$ is closed and convex. Suppose that $C_{k}$ is closed and convex for some $k \geq 1$. Next, we show that $C_{k+1}$ is closed and convex for some $k$. For any $p \in C_{k}$, we obtain that

$$
\left\|z_{k}-p\right\| \leq\left\|x_{k}-p\right\|
$$

is equivalent to

$$
\left\|z_{k}-x_{k}\right\|^{2}+2\left\langle z_{k}-x_{k}, x_{k}-p\right\rangle \leq 0
$$

Thus $C_{k+1}$ is closed and convex. Then, $C_{n}$ is closed and convex for any $\mathrm{n} \in \mathbb{N}$. This implies that $\left\{x_{n}\right\}$ is well defined.

Step 2. Next, we show by induction that $\Theta \subset C_{n}$ for each $n \geq 1$. 
Taking $p \in \Theta$ and by condition (ii), we get that $p=J_{M, \delta_{k}}\left(p-\delta_{k} B p\right)=J_{M, \lambda_{k}}\left(p-\lambda_{k} B p\right)$ is nonexpansive for all $n \geq 1$. From the assumption, we see that $\Theta \subset C=C_{1}$. Suppose $\Theta \subset C_{k}$ for some $k \geq 1$. For any $p \in \Theta=C_{k}$, we have

$$
\begin{aligned}
\left\|y_{k}-p\right\| & =\left\|J_{M, \delta_{k}}\left(u_{k}-\delta_{k} B u_{k}\right)-J_{M, \delta_{k}}\left(p-\delta_{k} B p\right)\right\| \\
& \leq\left\|\left(u_{k}-\delta_{k} B u_{k}\right)-\left(p-\delta_{k} B p\right)\right\| \\
& =\left\|\left(I-\delta_{k} B\right) u_{k}-\left(I-\delta_{k} B\right) p\right\| \\
& \leq\left\|u_{k}-p\right\| \leq\left\|x_{k}-p\right\|, \\
\left\|v_{k}-p\right\| & =\left\|J_{M, \lambda_{k}}\left(y_{k}-\lambda_{k} B y_{k}\right)-J_{M, \lambda_{k}}\left(p-\lambda_{k} B p\right)\right\| \\
& \leq\left\|\left(y_{k}-\lambda_{k} B y_{k}\right)-\left(p-\lambda_{k} B p\right)\right\| \\
& =\left\|\left(I-\lambda_{k} B\right) y_{k}-\left(I-\lambda_{k} B\right) p\right\| \\
& \leq\left\|y_{k}-p\right\| \leq\left\|x_{k}-p\right\| .
\end{aligned}
$$

Thus, we have

$$
\begin{aligned}
\left\|z_{k}-p\right\| & =\left\|\alpha_{k}\left(x_{k}-p\right)+\left(1-\alpha_{k}\right)\left(K_{k} v_{k}-p\right)\right\| \\
& \leq \alpha_{k}\left\|x_{k}-p\right\|+\left(1-\alpha_{k}\right)\left\|v_{k}-p\right\| \\
& \leq \alpha_{k}\left\|x_{k}-p\right\|+\left(1-\alpha_{k}\right)\left\|x_{k}-p\right\|=\left\|x_{k}-p\right\| .
\end{aligned}
$$

It follows that $p \in C_{k+1}$. This implies that $\Theta \subset C_{n}$ for each $n \geq 1$.

Step 3. Next, we show that $\lim _{n \rightarrow \infty}\left\|x_{n+1}-x_{n}\right\|=0$ and $\lim _{n \rightarrow \infty}\left\|x_{n}-z_{n}\right\|=0$.

From $x_{n}=P_{C_{n}} x_{0}$, we have

$$
\left\langle x_{0}-x_{n}, x_{n}-y\right\rangle \geq 0
$$

for each $y \in C_{n}$. Using $\Theta \subset C_{n}$, we also have

$$
\left\langle x_{0}-x_{n}, x_{n}-p\right\rangle \geq 0, \quad \forall p \in \Theta, n \in \mathbb{N} .
$$

So, for $p \in \Theta$, we have

$$
\begin{aligned}
0 & \leq\left\langle x_{0}-x_{n}, x_{n}-p\right\rangle \\
& =\left\langle x_{0}-x_{n}, x_{n}-x_{0}+x_{0}-p\right\rangle \\
& =-\left\langle x_{0}-x_{n}, x_{0}-x_{n}\right\rangle+\left\langle x_{0}-x_{n}, x_{0}-p\right\rangle \\
& \leq-\left\|x_{0}-x_{n}\right\|^{2}+\left\|x_{0}-x_{n}\right\|\left\|x_{0}-p\right\| .
\end{aligned}
$$


This implies that

$$
\left\|x_{0}-x_{n}\right\| \leq\left\|x_{0}-p\right\|, \quad \forall p \in \Theta, n \in \mathbb{N} .
$$

From $x_{n}=P_{C_{n}} x_{0}$ and $x_{n+1}=P_{C_{n+1}} x_{0} \in C_{n+1} \subset C_{n}$, we obtain

$$
\left\langle x_{0}-x_{n}, x_{n}-x_{n+1}\right\rangle \geq 0
$$

From (3.13), we have, for $n \in \mathbb{N}$,

$$
\begin{aligned}
0 & \leq\left\langle x_{0}-x_{n}, x_{n}-x_{n+1}\right\rangle \\
& =\left\langle x_{0}-x_{n}, x_{n}-x_{0}+x_{0}-x_{n+1}\right\rangle \\
& =-\left\langle x_{0}-x_{n}, x_{0}-x_{n}\right\rangle+\left\langle x_{0}-x_{n}, x_{0}-x_{n+1}\right\rangle \\
& \leq-\left\|x_{0}-x_{n}\right\|^{2}+\left\|x_{0}-x_{n}\right\|\left\|x_{0}-x_{n+1}\right\| .
\end{aligned}
$$

It follows that

$$
\left\|x_{0}-x_{n}\right\| \leq\left\|x_{0}-x_{n+1}\right\|
$$

Thus the sequence $\left\{\left\|x_{n}-x_{0}\right\|\right\}$ is a bounded and nonincreasing sequence, so $\lim _{n \rightarrow \infty}\left\|x_{n}-x_{0}\right\|$ exists, that is,

$$
\lim _{n \rightarrow \infty}\left\|x_{n}-x_{0}\right\|=m
$$

Indeed, from (3.13), we get

$$
\begin{aligned}
\left\|x_{n}-x_{n+1}\right\|^{2} & =\left\|x_{n}-x_{0}+x_{0}-x_{n+1}\right\|^{2} \\
& =\left\|x_{n}-x_{0}\right\|^{2}+2\left\langle x_{n}-x_{0}, x_{0}-x_{n+1}\right\rangle+\left\|x_{0}-x_{n+1}\right\|^{2} \\
& =\left\|x_{n}-x_{0}\right\|^{2}+2\left\langle x_{n}-x_{0}, x_{0}-x_{n}+x_{n}-x_{n+1}\right\rangle+\left\|x_{0}-x_{n+1}\right\|^{2} \\
& =\left\|x_{n}-x_{0}\right\|^{2}-2\left\langle x_{n}-x_{0}, x_{n}-x_{0}\right\rangle+2\left\langle x_{n}-x_{0}, x_{n}-x_{n+1}\right\rangle+\left\|x_{0}-x_{n+1}\right\|^{2} \\
& =-\left\|x_{n}-x_{0}\right\|^{2}+2\left\langle x_{n}-x_{0}, x_{n}-x_{n+1}\right\rangle+\left\|x_{0}-x_{n+1}\right\|^{2} \\
& \leq-\left\|x_{n}-x_{0}\right\|^{2}+\left\|x_{0}-x_{n+1}\right\|^{2} .
\end{aligned}
$$

From (3.16), we obtain

$$
\lim _{n \rightarrow \infty}\left\|x_{n}-x_{n+1}\right\|=0 .
$$


Since $x_{n+1}=P_{C_{n+1}} \in C_{n+1} \subset C_{n}$, we have

$$
\left\|x_{n}-z_{n}\right\| \leq\left\|x_{n}-x_{n+1}\right\|+\left\|x_{n+1}-z_{n}\right\| \leq 2\left\|x_{n}-x_{n+1}\right\| .
$$

By (3.18), we obtain

$$
\lim _{n \rightarrow \infty}\left\|x_{n}-z_{n}\right\|=0
$$

Step 4. Next, we show that $\lim _{n \rightarrow \infty}\left\|K_{n} v_{n}-v_{n}\right\|=0$.

For any given $p \in \Theta, \lambda_{n} \in(0,2 \xi]$. It is easy to see that $p=J_{M, \lambda_{n}}\left(p-\lambda_{n} B p\right)$. As $p-\lambda_{n} B p$ is nonexpansive, we have

$$
\begin{aligned}
\left\|v_{n}-p\right\|^{2} & =\left\|J_{M, \lambda_{n}}\left(y_{n}-\lambda_{n} B y_{n}\right)-J_{M, \lambda_{n}}\left(p-\lambda_{n} B p\right)\right\|^{2} \\
& \leq\left\|\left(y_{n}-\lambda_{n} B y_{n}\right)-\left(p-\lambda_{n} B p\right)\right\|^{2} \\
& =\left\|\left(y_{n}-p\right)-\lambda_{n}\left(B y_{n}-B p\right)\right\|^{2} \\
& =\left\|y_{n}-p\right\|^{2}-2 \lambda_{n}\left\langle y_{n}-p, B y_{n}-B p\right\rangle+\lambda_{n}^{2}\left\|B y_{n}-B p\right\|^{2} \\
& \leq\left\|x_{n}-p\right\|^{2}-2 \lambda_{n}\left\langle y_{n}-p, B y_{n}-B p\right\rangle+\lambda_{n}^{2}\left\|B y_{n}-B p\right\|^{2} \\
& \leq\left\|x_{n}-p\right\|^{2}+\lambda_{n}\left(\lambda_{n}-2 \xi\right)\left\|B y_{n}-B p\right\|^{2} .
\end{aligned}
$$

Similarly, we can prove that

$$
\left\|y_{n}-p\right\|^{2} \leq\left\|x_{n}-p\right\|^{2}+\delta_{n}\left(\delta_{n}-2 \xi\right)\left\|B u_{n}-B p\right\|^{2}
$$

Observe that

$$
\begin{aligned}
\left\|z_{n}-p\right\|^{2} & \leq \alpha_{n}\left\|x_{n}-p\right\|^{2}+\left(1-\alpha_{n}\right)\left\|K_{n} v_{n}-p\right\|^{2} \\
& \leq \alpha_{n}\left\|x_{n}-p\right\|^{2}+\left(1-\alpha_{n}\right)\left\|v_{n}-p\right\|^{2} .
\end{aligned}
$$

Substituting (3.21) into (3.23), and using conditions (i) and (ii), we have

$$
\begin{aligned}
\left\|z_{n}-p\right\|^{2} & \leq \alpha_{n}\left\|x_{n}-p\right\|^{2}+\left(1-\alpha_{n}\right)\left\|v_{n}-p\right\|^{2} \\
& \leq \alpha_{n}\left\|x_{n}-p\right\|^{2}+\left(1-\alpha_{n}\right)\left\{\left\|x_{n}-p\right\|^{2}+\lambda_{n}\left(\lambda_{n}-2 \xi\right)\left\|B y_{n}-B p\right\|^{2}\right\} \\
& =\left\|x_{n}-p\right\|^{2}+\left(1-\alpha_{n}\right) \lambda_{n}\left(\lambda_{n}-2 \xi\right)\left\|B y_{n}-B p\right\|^{2} .
\end{aligned}
$$


It follows that

$$
\begin{aligned}
(1-e) a(2 \xi-b)\left\|B y_{n}-B p\right\|^{2} & \leq\left(1-\alpha_{n}\right) \lambda_{n}\left(2 \xi-\lambda_{n}\right)\left\|B y_{n}-B p\right\|^{2} \\
& \leq\left\|x_{n}-p\right\|^{2}-\left\|z_{n}-p\right\|^{2} \\
& =\left(\left\|x_{n}-p\right\|-\left\|z_{n}-p\right\|\right)\left(\left\|x_{n}-p\right\|+\left\|z_{n}-p\right\|\right) \\
& \leq\left\|x_{n}-z_{n}\right\|\left(\left\|x_{n}-p\right\|+\left\|z_{n}-p\right\|\right) .
\end{aligned}
$$

Since $\lim _{n \rightarrow \infty}\left\|x_{n}-z_{n}\right\|=0$, we obtain

$$
\lim _{n \rightarrow \infty}\left\|B y_{n}-B p\right\|=0
$$

Since the resolvent operator $J_{M, \lambda_{n}}$ is 1-inverse strongly monotone, we obtain

$$
\begin{aligned}
\left\|v_{n}-p\right\|^{2}= & \left\|J_{M, \lambda_{n}}\left(y_{n}-\lambda_{n} B y_{n}\right)-J_{M, \lambda_{n}}\left(p-\lambda_{n} B p\right)\right\|^{2} \\
= & \left\|J_{M, \lambda_{n}}\left(I-\lambda_{n} B\right) y_{n}-J_{M, \lambda_{n}}\left(I-\lambda_{n} B\right) p\right\|^{2} \\
\leq & \left\langle\left(I-\lambda_{n} B\right) y_{n}-\left(I-\lambda_{n} B\right) p, v_{n}-p\right\rangle \\
= & \frac{1}{2}\left\{\left\|\left(I-\lambda_{n} B\right) y_{n}-\left(I-\lambda_{n} B\right) p\right\|^{2}+\left\|v_{n}-p\right\|^{2}\right. \\
& \left.\quad-\left\|\left(I-\lambda_{n} B\right) y_{n}-\left(I-\lambda_{n} B\right) p-\left(v_{n}-p\right)\right\|^{2}\right\} \\
\leq & \frac{1}{2}\left\{\left\|y_{n}-p\right\|^{2}+\left\|v_{n}-p\right\|^{2}-\left\|\left(y_{n}-v_{n}\right)-\lambda_{n}\left(B y_{n}-B p\right)\right\|^{2}\right\} \\
\leq & \frac{1}{2}\left\{\left\|x_{n}-p\right\|^{2}+\left\|v_{n}-p\right\|^{2}-\left\|y_{n}-v_{n}\right\|^{2}\right. \\
& \left.\quad-\lambda_{n}^{2}\left\|B y_{n}-B p\right\|^{2}+2 \lambda_{n}\left\langle y_{n}-v_{n}, B y_{n}-B p\right\rangle\right\},
\end{aligned}
$$

which yields that

$$
\left\|v_{n}-p\right\|^{2} \leq\left\|x_{n}-p\right\|^{2}-\left\|y_{n}-v_{n}\right\|^{2}+2 \lambda_{n}\left\|y_{n}-v_{n}\right\|\left\|B y_{n}-B p\right\|
$$

Similarly, we obtain

$$
\left\|y_{n}-p\right\|^{2} \leq\left\|x_{n}-p\right\|^{2}-\left\|u_{n}-y_{n}\right\|^{2}+2 \delta_{n}\left\|u_{n}-y_{n}\right\|\left\|B u_{n}-B p\right\| .
$$


Substituting (3.28) into (3.23), and using condition (i), we have

$$
\begin{aligned}
\left\|z_{n}-p\right\|^{2} & \leq \alpha_{n}\left\|x_{n}-p\right\|^{2}+\left(1-\alpha_{n}\right)\left\|v_{n}-p\right\|^{2} \\
& \leq \alpha_{n}\left\|x_{n}-p\right\|^{2}+\left(1-\alpha_{n}\right)\left\{\left\|x_{n}-p\right\|^{2}-\left\|y_{n}-v_{n}\right\|^{2}+2 \lambda_{n}\left\|y_{n}-v_{n}\right\|\left\|B y_{n}-B p\right\|\right\} \\
& =\left\|x_{n}-p\right\|^{2}-\left(1-\alpha_{n}\right)\left\|y_{n}-v_{n}\right\|^{2}+2\left(1-\alpha_{n}\right) \lambda_{n}\left\|y_{n}-v_{n}\right\|\left\|B y_{n}-B p\right\| .
\end{aligned}
$$

It follows that

$$
\begin{aligned}
\left(1-\alpha_{n}\right)\left\|y_{n}-v_{n}\right\|^{2} & \leq\left\|x_{n}-p\right\|^{2}-\left\|z_{n}-p\right\|^{2}+2\left(1-\alpha_{n}\right) \lambda_{n}\left\|y_{n}-v_{n}\right\|\left\|B y_{n}-B p\right\| \\
& \leq\left\|x_{n}-z_{n}\right\|\left(\left\|x_{n}-p\right\|+\left\|z_{n}-p\right\|\right)+2\left(1-\alpha_{n}\right) \lambda_{n}\left\|y_{n}-v_{n}\right\|\left\|B y_{n}-B p\right\|
\end{aligned}
$$

Applying $\left\|x_{n}-z_{n}\right\| \rightarrow 0$ and $\left\|\mathrm{B} y_{n}-B p\right\| \rightarrow 0$ as $n \rightarrow \infty$ to the last inequality, we get

$$
\lim _{n \rightarrow \infty}\left\|y_{n}-v_{n}\right\|=0
$$

Note that

$$
\begin{aligned}
\left\|z_{n}-p\right\|^{2} & \leq \alpha_{n}\left\|x_{n}-p\right\|^{2}+\left(1-\alpha_{n}\right)\left\|K_{n} v_{n}-p\right\|^{2} \\
& \leq \alpha_{n}\left\|x_{n}-p\right\|^{2}+\left(1-\alpha_{n}\right)\left\|v_{n}-p\right\|^{2} \\
& \leq \alpha_{n}\left\|x_{n}-p\right\|^{2}+\left(1-\alpha_{n}\right)\left\|y_{n}-p\right\|^{2} .
\end{aligned}
$$

Substituting (3.22) into (3.33), and using conditions (i) and (ii), we have

$$
\begin{aligned}
\left\|z_{n}-p\right\|^{2} & \leq \alpha_{n}\left\|x_{n}-p\right\|^{2}+\left(1-\alpha_{n}\right)\left\|y_{n}-p\right\|^{2} \\
& \leq \alpha_{n}\left\|x_{n}-p\right\|^{2}+\left(1-\alpha_{n}\right)\left\{\left\|x_{n}-p\right\|^{2}+\delta_{n}\left(\delta_{n}-2 \xi\right)\left\|B u_{n}-B p\right\|^{2}\right\} \\
& =\left\|x_{n}-p\right\|^{2}+\left(1-\alpha_{n}\right) \delta_{n}\left(\delta_{n}-2 \xi\right)\left\|B u_{n}-B p\right\|^{2} .
\end{aligned}
$$

It follows that

$$
\begin{aligned}
(1-e) a(2 \xi-b)\left\|B u_{n}-B p\right\|^{2} & \leq\left(1-\alpha_{n}\right) \delta_{n}\left(2 \xi-\delta_{n}\right)\left\|B u_{n}-B p\right\|^{2} \\
& \leq\left\|x_{n}-p\right\|^{2}-\left\|z_{n}-p\right\|^{2} \\
& \leq\left\|x_{n}-z_{n}\right\|\left(\left\|x_{n}-p\right\|+\left\|z_{n}-p\right\|\right) .
\end{aligned}
$$


Journal of Inequalities and Applications

Since $\lim _{n \rightarrow \infty}\left\|x_{n}-z_{n}\right\|=0$, we obtain

$$
\lim _{n \rightarrow \infty}\left\|B u_{n}-B p\right\|=0
$$

Substituting (3.29) into (3.33), and using conditions (i) and (ii), we have

$$
\begin{aligned}
\left\|z_{n}-p\right\|^{2} & \leq \alpha_{n}\left\|x_{n}-p\right\|^{2}+\left(1-\alpha_{n}\right)\left\|y_{n}-p\right\|^{2} \\
& \leq \alpha_{n}\left\|x_{n}-p\right\|^{2}+\left(1-\alpha_{n}\right)\left\{\left\|x_{n}-p\right\|^{2}-\left\|u_{n}-y_{n}\right\|^{2}+2 \delta_{n}\left\|u_{n}-y_{n}\right\|\left\|B u_{n}-B p\right\|\right\} \\
& =\left\|x_{n}-p\right\|^{2}-\left(1-\alpha_{n}\right)\left\|u_{n}-y_{n}\right\|^{2}+2\left(1-\alpha_{n}\right) \delta_{n}\left\|u_{n}-y_{n}\right\|\left\|B u_{n}-B p\right\| .
\end{aligned}
$$

It follows that

$$
\begin{aligned}
\left(1-\alpha_{n}\right)\left\|u_{n}-y_{n}\right\|^{2} & \leq\left\|x_{n}-p\right\|^{2}-\left\|z_{n}-p\right\|^{2}+2\left(1-\alpha_{n}\right) \delta_{n}\left\|u_{n}-y_{n}\right\|\left\|B u_{n}-B p\right\| \\
& \leq\left\|x_{n}-z_{n}\right\|\left(\left\|x_{n}-p\right\|+\left\|z_{n}-p\right\|\right)+2\left(1-\alpha_{n}\right) \delta_{n}\left\|u_{n}-y_{n}\right\|\left\|B u_{n}-B p\right\| .
\end{aligned}
$$

Applying $\left\|x_{n}-z_{n}\right\| \rightarrow 0$ and $\left\|B u_{n}-B p\right\| \rightarrow 0$ as $n \rightarrow \infty$ to the last inequality, we get

$$
\lim _{n \rightarrow \infty}\left\|u_{n}-y_{n}\right\|=0
$$

From (3.32) and (3.39), we have

$$
\lim _{n \rightarrow \infty}\left\|u_{n}-v_{n}\right\|=0 .
$$

From (3.33), (3.4), and condition (iii), we have

$$
\begin{aligned}
\left\|z_{n}-p\right\|^{2} & \leq \alpha_{n}\left\|x_{n}-p\right\|^{2}+\left(1-\alpha_{n}\right)\left\|y_{n}-p\right\|^{2} \\
& \leq \alpha_{n}\left\|x_{n}-p\right\|^{2}+\left(1-\alpha_{n}\right)\left\|u_{n}-p\right\|^{2} \\
& \leq \alpha_{n}\left\|x_{n}-p\right\|^{2}+\left(1-\alpha_{n}\right)\left\{\left\|x_{n}-p\right\|^{2}+r_{n}\left(r_{n}-2 \beta\right)\left\|A x_{n}-A p\right\|^{2}\right\} \\
& =\left\|x_{n}-p\right\|^{2}+\left(1-\alpha_{n}\right) r_{n}\left(r_{n}-2 \beta\right)\left\|A x_{n}-A p\right\|^{2} .
\end{aligned}
$$

It follows that

$$
\begin{aligned}
(1-e) c(2 \beta-d)\left\|A x_{n}-A p\right\|^{2} & \leq\left(1-\alpha_{n}\right) r_{n}\left(2 \beta-r_{n}\right)\left\|A x_{n}-A p\right\|^{2} \\
& \leq\left\|x_{n}-p\right\|^{2}-\left\|z_{n}-v\right\|^{2} \\
& \leq\left\|x_{n}-z_{n}\right\|\left(\left\|x_{n}-p\right\|+\left\|z_{n}-p\right\|\right) .
\end{aligned}
$$


Since $\lim _{n \rightarrow \infty}\left\|x_{n}-z_{n}\right\|=0$, we obtain

$$
\lim _{n \rightarrow \infty}\left\|A x_{n}-A p\right\|=0 .
$$

On the other hand, in the light of Lemma 2.6(3), $T_{r_{n}}$ is firmly nonexpansvie, so we have

$$
\begin{aligned}
\left\|u_{n}-p\right\|^{2}= & \left\|T_{r_{n}}\left(x_{n}-r_{n} A x_{n}\right)-T_{r_{n}}\left(p-r_{n} A p\right)\right\|^{2} \\
\leq & \left\langle T_{r_{n}}\left(x_{n}-r_{n} A x_{n}\right)-T_{r_{n}}\left(p-r_{n} A p\right), u_{n}-p\right\rangle \\
= & \left\langle x_{n}-r_{n} A x_{n}-\left(p-r_{n} A p\right), u_{n}-p\right\rangle \\
= & \frac{1}{2}\left\{\left\|\left(x_{n}-r_{n} A x_{n}\right)-\left(p-r_{n} A p\right)\right\|^{2}+\left\|u_{n}-p\right\|^{2}\right. \\
& \left.\quad-\left\|\left(x_{n}-r_{n} A x_{n}\right)-\left(p-r_{n} A p\right)-\left(u_{n}-p\right)\right\|^{2}\right\} \\
\leq & \frac{1}{2}\left\{\left\|x_{n}-p\right\|^{2}+\left\|u_{n}-p\right\|^{2}-\left\|x_{n}-p\right\|^{2}-r_{n}\left(A x_{n}-A p\right) \|^{2}\right\} \\
= & \frac{1}{2}\left\{\left\|x_{n}-p\right\|^{2}+\left\|u_{n}-p\right\|^{2}-\left\|x_{n}-u_{n}\right\|^{2}\right. \\
& \left.\quad+2 r_{n}\left\langle x_{n}-u_{n}, A x_{n}-A p\right\rangle-r_{n}^{2}\left\|A x_{n}-A p\right\|^{2}\right\},
\end{aligned}
$$

which implies that

$$
\left\|u_{n}-p\right\|^{2} \leq\left\|x_{n}-p\right\|^{2}-\left\|x_{n}-u_{n}\right\|^{2}+2 r_{n}\left\|x_{n}-u_{n}\right\|\left\|A x_{n}-A p\right\| .
$$

Using (3.41) again and (3.45), we have

$$
\begin{aligned}
\left\|z_{n}-p\right\|^{2} & \leq \alpha_{n}\left\|x_{n}-p\right\|^{2}+\left(1-\alpha_{n}\right)\left\|u_{n}-p\right\|^{2} \\
& \leq \alpha_{n}\left\|x_{n}-p\right\|^{2}+\left(1-\alpha_{n}\right)\left\{\left\|x_{n}-p\right\|^{2}-\left\|x_{n}-u_{n}\right\|^{2}+2 r_{n}\left\|x_{n}-u_{n}\right\|\left\|A x_{n}-A p\right\|\right\} \\
& =\left\|x_{n}-p\right\|^{2}-\left(1-\alpha_{n}\right)\left\|x_{n}-u_{n}\right\|^{2}+2\left(1-\alpha_{n}\right) r_{n}\left\|x_{n}-u_{n}\right\|\left\|A x_{n}-A p\right\| .
\end{aligned}
$$

It follows from the condition (i) that

$$
\begin{aligned}
(1-e)\left\|x_{n}-u_{n}\right\|^{2} & \leq\left(1-\alpha_{n}\right)\left\|x_{n}-u_{n}\right\|^{2} \\
& \leq\left\|x_{n}-p\right\|^{2}-\left\|z_{n}-p\right\|^{2}+2\left(1-\alpha_{n}\right) r_{n}\left\|x_{n}-u_{n}\right\|\left\|A x_{n}-A p\right\| \\
& \leq\left\|x_{n}-z_{n}\right\|\left(\left\|x_{n}-p\right\|+\left\|z_{n}-p\right\|\right)+2\left(1-\alpha_{n}\right) r_{n}\left\|x_{n}-u_{n}\right\|\left\|A x_{n}-A p\right\| .
\end{aligned}
$$


Since $\lim _{n \rightarrow \infty}\left\|x_{n}-z_{n}\right\|=0$ and $\lim _{n \rightarrow \infty}\left\|A x_{n}-A p\right\|=0$, it is implied that

$$
\lim _{n \rightarrow \infty}\left\|x_{n}-u_{n}\right\|=0
$$

From (3.39) and (3.48), we have

$$
\lim _{n \rightarrow \infty}\left\|x_{n}-y_{n}\right\|=0
$$

By (3.3), we get

$$
z_{n}-x_{n}=\left(1-\alpha_{n}\right)\left(K_{n} v_{n}-x_{n}\right)
$$

Since $\left\{\alpha_{n}\right\} \subset[0, e]$ for some $e$ with $0 \leq e<1$, and $\left\|x_{n}-z_{n}\right\| \rightarrow \infty$ as $n \rightarrow \infty$, we also have

$$
\lim _{n \rightarrow \infty}\left\|K_{n} v_{n}-x_{n}\right\|=0
$$

From (3.40) and (3.48), we have

$$
\lim _{n \rightarrow \infty}\left\|x_{n}-v_{n}\right\|=0
$$

Furthermore, by the triangular inequality, we also have

$$
\left\|K_{n} v_{n}-v_{n}\right\| \leq\left\|K_{n} v_{n}-x_{n}\right\|+\left\|x_{n}-v_{n}\right\| .
$$

Applying (3.51) and (3.52), we obtain

$$
\lim _{n \rightarrow \infty}\left\|K_{n} v_{n}-v_{n}\right\|=0
$$

Let $K$ be the mapping defined by (3.1). Since $\left\{v_{n}\right\}$ is bounded, applying Lemma 3.2 and (3.54), we have

$$
\left\|K v_{n}-v_{n}\right\| \leq\left\|K v_{n}-K_{n} v_{n}\right\|+\left\|K_{n} v_{n}-v_{n}\right\| \longrightarrow 0 \quad \text { as } n \longrightarrow \infty .
$$

Step 5. Next, we show that $q \in \Theta:=\cap_{i=1}^{N} F\left(T_{i}\right) \cap \operatorname{GMEP}(F, \varphi, A) \cap I(B, M)$.

Since $\left\{v_{n}\right\}$ is bounded, there exists a subsequence $\left\{v_{n_{i}}\right\}$ of $\left\{v_{n}\right\}$ which converges weakly to $q$. Without loss of generality, we can assume that $v_{n_{i}} \rightarrow q$. Since $v_{n_{i}} \subset C$ and $C$ is closed and convex, $C$ is weakly closed and hence $q \in C$. From $\left\|K v_{n}-v_{n}\right\| \rightarrow 0$, we obtain $K v_{n_{i}} \rightarrow q$.

(a) First, we prove that $q \in I(B, M)$.

We observe that $B$ is a $1 / \xi$-Lipschitz monotone mapping and $D(B)=H$. From Lemma 2.2, we know that $M+B$ is maximal monotone. Let $(v, g) \in G(M+B)$, that is, $g-B v \in M(v)$. Since $v_{n_{i}}=J_{M, \lambda_{n_{i}}}\left(y_{n_{i}}-\lambda_{n_{i}} B y_{n_{i}}\right)$, we have

$$
y_{n_{i}}-\lambda_{n_{i}} B y_{n_{i}} \in\left(I+\lambda_{n_{i}} M\right)\left(v_{n_{i}}\right)
$$


that is,

$$
\frac{1}{\lambda_{n_{i}}}\left(y_{n_{i}}-v_{n_{i}}-\lambda_{n_{i}} B y_{n_{i}}\right) \in M\left(v_{n_{i}}\right) .
$$

By virtue of the maximal monotonicity of $M+B$, we have

$$
\left\langle v-v_{n_{i}}, g-B v-\frac{1}{\lambda_{n_{i}}}\left(y_{n_{i}}-v_{n_{i}}-\lambda_{n_{i}} B y_{n_{i}}\right)\right\rangle \geq 0,
$$

and so

$$
\begin{aligned}
\left\langle v-v_{n_{i}}, g\right\rangle & \geq\left\langle v-v_{n_{i}}, B v+\frac{1}{\lambda_{n_{i}}}\left(y_{n_{i}}-v_{n_{i}}-\lambda_{n_{i}} B y_{n_{i}}\right)\right\rangle \\
& =\left\langle v-v_{n_{i}}, B v-B z_{n}+B v_{n_{i}}-B y_{n_{i}}+\frac{1}{\lambda_{n_{i}}}\left(y_{n_{i}}-v_{n_{i}}\right)\right\rangle \\
& \geq 0+\left\langle v-v_{n_{i}}, B v_{n_{i}}-B y_{n_{i}}\right\rangle+\left\langle v-v_{n_{i}}, \frac{1}{\lambda_{n_{i}}}\left(y_{n_{i}}-v_{n_{i}}\right)\right\rangle .
\end{aligned}
$$

It follows from $\left\|y_{n}-v_{n}\right\| \rightarrow 0,\left\|B y_{n}-B v_{n}\right\| \rightarrow 0$ and $v_{n_{i}} \rightarrow q$ that

$$
\lim _{n \rightarrow \infty}\left\langle v-v_{n_{i}}, g\right\rangle=\langle v-q, g\rangle \geq 0
$$

It follows from the maximal monotonicity of $M+B$ that $\theta \in(M+B)(q)$, that is, $q \in I(B, M)$. have

(b) Next, we show that $q \in \operatorname{GMEP}(F, \varphi, A)$. Since $u_{n}=T_{r_{n}}\left(x_{n}-r_{n} A x_{n}\right) \in \operatorname{dom} \varphi$, we

$$
F\left(u_{n}, y\right)+\left\langle A x_{n}, y-u_{n}\right\rangle+\varphi(y)-\varphi\left(u_{n}\right)+\frac{1}{r_{n}}\left\langle y-u_{n}, u_{n}-x_{n}\right\rangle \geq 0, \quad \forall y \in C
$$

From (A2), we also have

$$
\left\langle A x_{n}, y-u_{n}\right\rangle+\varphi(y)-\varphi\left(u_{n}\right)+\frac{1}{r_{n}}\left\langle y-u_{n}, u_{n}-x_{n}\right\rangle \geq F\left(y, u_{n}\right), \quad \forall y \in C .
$$

And hence

$$
\left\langle A x_{n_{i}}, y-u_{n_{i}}\right\rangle+\varphi(y)-\varphi\left(u_{n_{i}}\right)+\left\langle y-u_{n_{i}}, \frac{u_{n_{i}}-x_{n_{i}}}{r_{n_{i}}}\right\rangle \geq F\left(y, u_{n_{i}}\right), \quad \forall y \in C .
$$


For $t$ with $0<t \leq 1$ and $y \in C$, let $y_{t}=t y+(1-t) q$. Since $y \in C$ and $q \in C$, we have $y_{t} \in C$. So, from (3.63), we have

$$
\begin{aligned}
\left\langle y_{t}-u_{n_{i}}, A y_{t}\right\rangle \geq & \left\langle y_{t}-u_{n_{i}}, A y_{t}\right\rangle-\varphi\left(y_{t}\right)+\varphi\left(u_{n_{i}}\right)-\left\langle y_{t}-u_{n_{i}}, A x_{n_{i}}\right\rangle \\
& -\left\langle y_{t}-u_{n_{i}}, \frac{u_{n_{i}}-x_{n_{i}}}{r_{n_{i}}}\right\rangle+F\left(y_{t}, u_{n_{i}}\right) \\
= & \left\langle y_{t}-u_{n_{i}}, A y_{t}-A u_{n_{i}}\right\rangle+\left\langle y_{t}-u_{n_{i}}, A u_{n_{i}}-A x_{n_{i}}\right\rangle-\varphi\left(y_{t}\right)+\varphi\left(u_{n_{i}}\right) \\
& -\left\langle y_{t}-u_{n_{i}}, \frac{u_{n_{i}}-x_{n_{i}}}{r_{n_{i}}}\right\rangle+F\left(y_{t}, u_{n_{i}}\right) .
\end{aligned}
$$

Since $\left\|u_{n_{i}}-x_{n_{i}}\right\| \rightarrow 0$, we have $\left\|A u_{n_{i}}-A x_{n_{i}}\right\| \rightarrow 0$. Further, from the inverse strongly monotonicity of $A$, we have $\left\langle y_{t}-u_{n_{i}}, A y_{t}-A u_{n_{i}}\right\rangle \geq 0$. So, from (A5), the weakly lower semicontinuity of $\varphi,\left(u_{n_{i}}-x_{n_{i}}\right) / r_{n_{i}} \rightarrow 0$ and $u_{n_{i}} \rightarrow q$, we have

$$
\left\langle y_{t}-q, A y_{t}\right\rangle \geq-\varphi\left(y_{t}\right)+\varphi(q)+F\left(y_{t}, q\right)
$$

as $i \rightarrow \infty$. From (A1), (A4) and (3.65), we also get

$$
\begin{aligned}
0 & =F\left(y_{t}, y_{t}\right)+\varphi\left(y_{t}\right)-\varphi\left(y_{t}\right) \\
& \leq t F\left(y_{t}, y\right)+(1-t) F\left(y_{t}, q\right)+t \varphi(y)-(1-t) \varphi(q)-\varphi\left(y_{t}\right) \\
& =t\left[F\left(y_{t}, y\right)+\varphi(y)-\varphi\left(y_{t}\right)\right]+(1-t)\left[F\left(y_{t}, q\right)+\varphi(q)-\varphi\left(y_{t}\right)\right] \\
& \leq t\left[F\left(y_{t}, y\right)+\varphi(y)-\varphi\left(y_{t}\right)\right]+(1-t)\left\langle y_{t}-q, A y_{t}\right\rangle \\
& =t\left[F\left(y_{t}, y\right)+\varphi(y)-\varphi\left(y_{t}\right)\right]+(1-t) t\left\langle y-q, A y_{t}\right\rangle, \\
& \leq F\left(y_{t}, y\right)+\varphi(y)-\varphi\left(y_{t}\right)+(1-t)\left\langle y-q, A y_{t}\right\rangle .
\end{aligned}
$$

Letting $t \rightarrow 0$, we have, for each $y \in C$,

$$
F(q, y)+\varphi(y)-\varphi(q)+\langle y-q, A q\rangle \geq 0 .
$$

This implies that $q \in \operatorname{GMEP}(F, \varphi, A)$.

(c) Now, we prove that $q \in F(K)=\bigcap_{i=1}^{N} F\left(T_{i}\right)$. 
Assume $q \notin F(K)$. Since $\left\|x_{n}-v_{n}\right\| \rightarrow 0$ and we know that $v_{n_{i}} \rightarrow q(i \rightarrow \infty)$ and $q \neq K q$, it follows by the Opial's condition (Lemma 2.4) that

$$
\begin{aligned}
\liminf _{i \rightarrow \infty}\left\|v_{n_{i}}-q\right\| & <\liminf _{i \rightarrow \infty}\left\|v_{n_{i}}-K q\right\| \\
& \leq \liminf _{i \rightarrow \infty}\left(\left\|v_{n_{i}}-K v_{n_{i}}\right\|+\left\|K v_{n_{i}}-K q\right\|\right) \\
& <\liminf _{i \rightarrow \infty}\left\|v_{n_{i}}-q\right\|,
\end{aligned}
$$

which is a contradiction. Thus, we get $q \in F(\mathrm{~K})=\bigcap_{i=1}^{N} F\left(T_{i}\right)$.

The conclusion is $q \in \Theta:=\cap_{i=1}^{N} F\left(T_{i}\right) \cap \operatorname{GMEP}(F, \varphi, A) \cap I(B, M)$.

Step 6. Finally, we show that $x_{n} \rightarrow z$ and $u_{n} \rightarrow z$, where $z=P_{\Theta} x_{0}$.

Since $\Theta$ is nonempty closed convex subset of $H$, there exists a unique $z^{\prime} \in \Theta$ such that $z^{\prime}=P_{\Theta} x_{0}$. Since $z^{\prime} \in \Theta \subset C_{n}$ and $x_{n}=P_{C_{n}} x_{0}$, we have

$$
\left\|x_{0}-x_{n}\right\|=\left\|x_{0}-P_{C_{n}} x_{0}\right\| \leq\left\|x_{0}-z^{\prime}\right\|
$$

for all $n \in \mathbb{N}$. From (3.69), $\left\{x_{n}\right\}$ is bounded, so $\omega_{w}\left(x_{n}\right) \neq \emptyset$. By the weak lower semicontinuity of the norm, we have

$$
\left\|x_{0}-z\right\| \leq \liminf _{i \rightarrow \infty}\left\|x_{0}-x_{n_{i}}\right\| \leq\left\|x_{0}-z^{\prime}\right\|
$$

However, Since $z \in \omega_{w}\left(x_{n}\right) \subset \Theta$, we have

$$
\left\|x_{0}-z^{\prime}\right\| \leq\left\|x_{0}-P_{C_{n}} x_{0}\right\| \leq\left\|x_{0}-z\right\| .
$$

Using (3.69) and (3.70), we obtain $z^{\prime}=z$. Thus $\omega_{w}\left(x_{n}\right)=\{z\}$ and $x_{n} \rightarrow z$. So, we have

$$
\left\|x_{0}-z^{\prime}\right\| \leq\left\|x_{0}-z\right\| \leq \liminf _{n \rightarrow \infty}\left\|x_{0}-x_{n}\right\| \leq \limsup _{n \rightarrow \infty}\left\|x_{0}-x_{n}\right\| \leq\left\|x_{0}-z^{\prime}\right\| .
$$

Thus, we obtain that

$$
\left\|x_{0}-z\right\|=\lim _{n \rightarrow \infty}\left\|x_{0}-x_{n}\right\|=\left\|x_{0}-z^{\prime}\right\|
$$

From $x_{n} \rightarrow z$, we obtain $\left(x_{0}-x_{n}\right) \rightarrow\left(x_{0}-z\right)$. Using the Kadec-Klee property (Lemma 2.5) of $H$, we obtain that

$$
\left\|x_{n}-z\right\|=\left\|\left(x_{n}-x_{0}\right)-\left(z-x_{0}\right)\right\| \longrightarrow 0 \quad \text { as } n \longrightarrow \infty
$$

and hence $x_{n} \rightarrow z$ in norm. Finally, noticing $\left\|u_{n}-z\right\|=\left\|T_{r_{n}}\left(x_{n}-r_{n} A x_{n}\right)-T_{r_{n}}\left(z-r_{n} A z\right)\right\| \leq$ $\left\|x_{n}-z\right\|$. we also conclude that $u_{n} \rightarrow z$ in norm. This completes the proof. 
Corollary 3.4. Let $C$ be a nonempty closed convex subset of a real Hilbert space $H$, let $F$ be a bifunction from $C \times C$ to $\mathbb{R}$ satisfying (A1)-(A4), and let $\varphi: C \rightarrow \mathbb{R} \cup\{+\infty\}$ be a proper lower semicontinuous and convex function with assumption (B1) or (B2). Let $\left\{T_{i}\right\}_{i=1}^{N}$ be a finite family of nonexpansive mappings of $C$ into itself, let $A$ be a $\beta$-inverse-strongly monotone mapping of $C$ into $H$, let $B$ be a $\xi$-inverse-strongly monotone mapping of $C$ into $H$ and $M: H \rightarrow 2^{H}$ a maximal monotone mapping. Assume that

$$
\Theta:=\bigcap_{i=1}^{N} F\left(T_{i}\right) \cap \operatorname{GMEP}(F, \varphi, A) \cap I(B, M) \neq \emptyset
$$

Let $K_{n}$ be the K-mapping generated by $T_{1}, T_{2}, \ldots, T_{N}$ and $\gamma_{n, 1}, \gamma_{n, 2}, \ldots, \gamma_{n, N}$. Let $\left\{x_{n}\right\},\left\{y_{n}\right\},\left\{v_{n}\right\}$, $\left\{z_{n}\right\}$ and $\left\{u_{n}\right\}$ be sequences generated by (3.3) satisfying the following conditions in Theorem 3.3. Then, $\left\{x_{n}\right\}$ and $\left\{u_{n}\right\}$ converge strongly to $z=P_{\Theta} x_{0}$.

From Theorem 3.3, we can obtain the following results.

Theorem 3.5. Let $C$ be a nonempty closed convex subset of a real Hilbert space $H$, let $F$ be a bifunction from $C \times C$ to $\mathbb{R}$ satisfying (A1)-(A4), and let $\varphi: C \rightarrow \mathbb{R} \cup\{+\infty\}$ be a proper lower semicontinuous and convex function with assumption (B1) or (B2). Let $\left\{T_{i}\right\}_{i=1}^{N}$ be a finite family of quasi-nonexpansive and $L_{i}$-Lipschitz mappings of $C$ into itself, let $A$ be a $\beta$-inverse-strongly monotone mapping of $C$ into $H$ and let $B$ be a $\xi$-inverse-strongly monotone mapping of $C$ into $H$. Assume that

$$
\Theta:=\bigcap_{i=1}^{N} F\left(T_{i}\right) \cap \operatorname{GMEP}(F, \varphi, A) \cap V I(C, B) \neq \emptyset
$$

Let $K_{n}$ be the K-mapping generated by $T_{1}, T_{2}, \ldots, T_{N}$ and $\gamma_{n, 1}, \gamma_{n, 2}, \ldots, \gamma_{n, N}$. Let $\left\{x_{n}\right\},\left\{y_{n}\right\},\left\{v_{n}\right\}$, $\left\{z_{n}\right\}$ and $\left\{u_{n}\right\}$ be sequences generated by $x_{0} \in H, C_{1}=C, x_{1}=P_{C_{1}} x_{0}, u_{n} \in C$ and let

$$
\begin{gathered}
F\left(u_{n}, y\right)+\varphi(y)-\varphi\left(u_{n}\right)+\left\langle A x_{n}, y-u_{n}\right\rangle+\frac{1}{r_{n}}\left\langle y-u_{n}, u_{n}-x_{n}\right\rangle \geq 0, \quad \forall y \in C, \\
y_{n}=P_{C}\left(u_{n}-\delta_{n} B u_{n}\right), \\
v_{n}=P_{C}\left(y_{n}-\lambda_{n} B y_{n}\right), \\
z_{n}=\alpha_{n} x_{n}+\left(1-\alpha_{n}\right) K_{n} v_{n}, \\
C_{n+1}=\left\{z \in C_{n}:\left\|z_{n}-z\right\| \leq\left\|x_{n}-z\right\|\right\}, \\
x_{n+1}=P_{C_{n+1}} x_{0}, \quad n \in \mathbb{N},
\end{gathered}
$$

where $\left\{\alpha_{n}\right\} \subset(0,1)$ satisfy the following conditions:

(i) $\left\{\alpha_{n}\right\} \subset[0, e]$ for some e with $0 \leq e<1$;

(ii) $\left\{\delta_{n}\right\},\left\{\lambda_{n}\right\} \subset[a, b]$ for some $a, b$ with $0<a<b<2 \xi$;

(iii) $\left\{r_{n}\right\} \subset[c, d]$ for some $c, d$ with $0<c<d<2 \beta$.

Then, $\left\{x_{n}\right\}$ and $\left\{u_{n}\right\}$ converge strongly to $z=P_{\Theta} x_{0}$. 
Proof. In Theorem 3.3 take $M=\partial \delta_{C}: H \rightarrow 2^{H}$, where $\delta_{C}: 0 \rightarrow[0, \infty]$ is the indicator function of $C$, that is,

$$
\delta_{C}(x)= \begin{cases}0, & x \in C, \\ +\infty, & x \notin C .\end{cases}
$$

Then the variational inclusion problem (1.3) is equivalent to variational inequality problem (1.14), that is, to find $\hat{x} \in C$ such that

$$
\langle B \widehat{x}, y-\widehat{x}\rangle \geq 0, \quad \forall y \in C
$$

Again, since $M=\delta_{C}$, then

$$
J_{M, \lambda_{n}}=J_{M, \delta_{n}}=P_{C},
$$

and so we have

$$
\begin{gathered}
y_{n}=P_{C}\left(u_{n}-\delta_{n} B u_{n}\right)=J_{M, \delta_{n}}\left(P_{C}\left(u_{n}-\delta_{n} B u_{n}\right)\right), \\
v_{n}=P_{C}\left(y_{n}-\lambda_{n} B y_{n}\right)=J_{M, \lambda_{n}}\left(P_{C}\left(y_{n}-\lambda_{n} B y_{n}\right)\right) .
\end{gathered}
$$

We can obtain the desired conclusion from Theorem 3.3 immediately. tractions.

Next, we consider another class of important nonlinear mappings: strict pseudocon-

Definition 3.6. A mapping $S: C \rightarrow C$ is called strictly pseudocontraction if there exists a constant $0 \leq \kappa<1$ such that

$$
\|S x-S y\|^{2} \leq\|x-y\|^{2}+\kappa\|(I-S) x-(I-S) y\|^{2}, \quad \forall x, y \in C .
$$

If $\kappa=0$, then $S$ is nonexpansive.

In this case, let $S: C \rightarrow C$ a $\mathcal{K}$-strictly pseudocontraction. Putting $B=I-S: C \rightarrow H$, then $B$ is a $(1-\kappa) / 2$-inverse-strongly monotone mapping. In fact, from (3.82) we have

$$
\|(I-B) x-(I-B) y\|^{2} \leq\|x-y\|^{2}+\kappa\|B x-B y\|^{2}, \quad \forall x, y \in C .
$$

Observe that

$$
\|(I-B) x-(I-B) y\|^{2}=\|x-y\|^{2}-2\langle x-y, B x-B y\rangle+\|B x-B y\|^{2}, \quad \forall x, y \in C .
$$


Hence, we obtain

$$
\langle x-y, B x-B y\rangle \geq \frac{1-\kappa}{2}\|B x-B y\|^{2}, \quad \forall x, y \in C .
$$

This shows that $B$ is $(1-\kappa) / 2$-inverse-strongly monotone mapping.

Now, we get the following result.

Theorem 3.7. Let $C$ be a nonempty closed convex subset of a real Hilbert space $H$, let $F$ be a bifunction from $C \times C$ to $\mathbb{R}$ satisfying $(A 1)-(A 4)$ and let $\varphi: C \rightarrow \mathbb{R} \cup\{+\infty\}$ be a proper lower semicontinuous and convex function with assumption (B1) or (B2). Let $\left\{T_{i}\right\}_{i=1}^{N}$ be a finite family of quasi-nonexpansive and $L_{i}$-Lipschitz mappings of $C$ into itself, let $S_{A}$ be a $\kappa_{\beta}$-strictly pseudocontraction mapping of $C$ into $C$ and let $S_{B}$ be a $\kappa_{\xi}$-strictly pseudocontraction mapping of $C$ into $C$. Assume that

$$
\Theta:=\bigcap_{i=1}^{N} F\left(T_{i}\right) \cap \operatorname{GMEP}\left(F, \varphi, I-S_{A}\right) \cap F\left(S_{B}\right) \neq \emptyset
$$

Let $K_{n}$ be the K-mapping generated by $T_{1}, T_{2}, \ldots, T_{N}$ and $\gamma_{n, 1}, \gamma_{n, 2}, \ldots, \gamma_{n, N}$. Let $\left\{x_{n}\right\},\left\{y_{n}\right\},\left\{v_{n}\right\}$, $\left\{z_{n}\right\}$ and $\left\{u_{n}\right\}$ be sequences generated by $x_{0} \in H, C_{1}=C, x_{1}=P_{C_{1}} x_{0}, u_{n} \in C$ and let

$$
\begin{gathered}
F\left(u_{n}, y\right)+\varphi(y)-\varphi\left(u_{n}\right)+\left\langle\left(I-S_{A}\right) x_{n}, y-u_{n}\right\rangle+\frac{1}{r_{n}}\left\langle y-u_{n}, u_{n}-x_{n}\right\rangle \geq 0, \quad \forall y \in C, \\
y_{n}=\left(1-\delta_{n}\right) u_{n}+\delta_{n} S_{B} u_{n}, \\
v_{n}=\left(1-\lambda_{n}\right) y_{n}+\lambda_{n} S_{B} y_{n}, \\
z_{n}=\alpha_{n} x_{n}+\left(1-\alpha_{n}\right) K_{n} v_{n} \\
C_{n+1}=\left\{z \in C_{n}:\left\|z_{n}-z\right\| \leq\left\|x_{n}-z\right\|\right\}, \\
x_{n+1}=P_{C_{n+1}} x_{0}, \quad n \in \mathbb{N},
\end{gathered}
$$

where $\left\{\alpha_{n}\right\} \subset(0,1)$ satisfy the following conditions:

(i) $\left\{\alpha_{n}\right\} \subset[0, e]$ for some $e$ with $0 \leq e<1$;

(ii) $\left\{\delta_{n}\right\},\left\{\lambda_{n}\right\} \subset[a, b]$ for some $a, b$ with $0<a<b<1-\kappa_{\xi}$;

(iii) $\left\{r_{n}\right\} \subset[c, d]$ for some $c, d$ with $0<c<d<1-\kappa_{\beta}$.

Then, $\left\{x_{n}\right\}$ and $\left\{u_{n}\right\}$ converge strongly to $P_{\Theta} x_{0}$.

Proof. Taking $A=I-S_{A}$ and $B=I-S_{B}$, respectively. Then we see that $A$ is $\left(1-\kappa_{\beta}\right) / 2-$ inverse-strongly monotone and $B$ is $\left(1-\kappa_{\xi}\right) / 2$-inverse-strongly monotone, respectively. We have $F\left(S_{B}\right)=\operatorname{VI}(C, B)$ and

$$
\begin{gathered}
y_{n}=P_{C}\left(u_{n}-\delta_{n} B u_{n}\right)=P_{C}\left(\left(1-\delta_{n}\right) u_{n}+\delta_{n} S_{B} u_{n}\right)=\left(1-\delta_{n}\right) u_{n}+\delta_{n} S_{B} u_{n} \in C, \\
v_{n}=P_{C}\left(y_{n}-\lambda_{n} B y_{n}\right)=P_{C}\left(\left(1-\lambda_{n}\right) y_{n}+\lambda_{n} S_{B} y_{n}\right)=\left(1-\lambda_{n}\right) y_{n}+\lambda_{n} S_{B} y_{n} \in C .
\end{gathered}
$$

By using Theorem 3.5, it is easy to obtain the desired conclusion. 


\section{Acknowledgments}

The authors would like to express their thank to the referees for helpful suggestions. The first author was supported by the National Research Council of Thailand and the Faculty of Science and Technology RMUTT Research Fund. The second author was supported by Rajamangala University of Technology Rattanakosin Research and Development Institute. The third author was supported by the Thailand Research Fund and the Commission on Higher Education under Grant No. MRG5380044.

\section{References}

[1] H. Brézis, "Opérateur maximaux monotones," in Mathematics Studies, vol. 5, North-Holland, Amsterdam, The Netherlands, 1973.

[2] B. Lemaire, "Which fixed point does the iteration method select?" in Recent Advances in Optimization (Trier, 1996), vol. 452 of Lecture Notes in Economics and Mathematical Systems, pp. 154-167, Springer, Berlin, Germany, 1997.

[3] E. Blum and W. Oettli, "From optimization and variational inequalities to equilibrium problems," The Mathematics Student, vol. 63, no. 1-4, pp. 123-145, 1994.

[4] O. Chadli, S. Schaible, and J. C. Yao, "Regularized equilibrium problems with application to noncoercive hemivariational inequalities," Journal of Optimization Theory and Applications, vol. 121, no. 3, pp. 571-596, 2004.

[5] O. Chadli, N. C. Wong, and J. C. Yao, "Equilibrium problems with applications to eigenvalue problems," Journal of Optimization Theory and Applications, vol. 117, no. 2, pp. 245-266, 2003.

[6] I. V. Konnov, S. Schaible, and J. C. Yao, "Combined relaxation method for mixed equilibrium problems," Journal of Optimization Theory and Applications, vol. 126, no. 2, pp. 309-322, 2005.

[7] A. Moudafi and M. Théra, "Proximal and dynamical approaches to equilibrium problems," in Ill-Posed Variational Problems and Regularization Techniques (Trier, 1998), vol. 477 of Lecture Notes in Economics and Mathematical Systems, pp. 187-201, Springer, Berlin, Germany, 1999.

[8] L.-C. Zeng, S.-Y. Wu, and J.-C. Yao, "Generalized KKM theorem with applications to generalized minimax inequalities and generalized equilibrium problems," Taiwanese Journal of Mathematics, vol. 10, no. 6, pp. 1497-1514, 2006.

[9] L. C. Ceng, D. R. Sahu, and J. C. Yao, "Implicit iterative algorithms for asymptotically nonexpansive mappings in the intermediate sense and Lipschitz-continuous monotone mappings," Journal of Computational and Applied Mathematics, vol. 233, no. 11, pp. 2902-2915, 2010.

[10] L.-C. Ceng and J.-C. Yao, "A relaxed extragradient-like method for a generalized mixed equilibrium problem, a general system of generalized equilibria and a fixed point problem," Nonlinear Analysis: Theory, Methods E Applications, vol. 72, no. 3-4, pp. 1922-1937, 2010.

[11] L. C. Ceng, A. Petruşel, and J. C. Yao, "Iterative approaches to solving equilibrium problems and fixed point problems of infinitely many nonexpansive mappings," Journal of Optimization Theory and Applications, vol. 143, no. 1, pp. 37-58, 2009.

[12] F. Cianciaruso, G. Marino, L. Muglia, and Y. Yao, "A hybrid projection algorithm for finding solutions of mixed equilibrium problem and variational inequality problem," Fixed Point Theory and Applications, vol. 2010, Article ID 383740, 19 pages, 2010.

[13] O. Chadli, Z. Liu, and J. C. Yao, "Applications of equilibrium problems to a class of noncoercive variational inequalities," Journal of Optimization Theory and Applications, vol. 132, no. 1, pp. 89-110, 2007.

[14] P. Cholamjiak and S. Suantai, "A new hybrid algorithm for variational inclusions, generalized equilibrium problems, and a finite family of quasi-nonexpansive mappings," Fixed Point Theory and Applications, vol. 2009, Article ID 350979, 20 pages, 2009.

[15] C. Jaiboon and P. Kumam, "A general iterative method for addressing mixed equilibrium problems and optimization problems," Nonlinear Analysis, Theory, Methods and Applications, vol. 73, pp. 1180 1202, 2010.

[16] C. Jaiboon and P. Kumam, "Strong convergence for generalized equilibrium problems, fixed point problems and relaxed cocoercive variational inequalities," Journal of Inequalities and Applications, vol. 2010, Article ID 728028, 43 pages, 2010. 
[17] C. Jaiboon, W. Chantarangsi, and P. Kumam, "A convergence theorem based on a hybrid relaxed extragradient method for generalized equilibrium problems and fixed point problems of a finite family of nonexpansive mappings," Nonlinear Analysis: Hybrid Systems, vol. 4, no. 1, pp. 199-215, 2010.

[18] P. Kumam and C. Jaiboon, "A new hybrid iterative method for mixed equilibrium problems and variational inequality problem for relaxed cocoercive mappings with application to optimization problems," Nonlinear Analysis: Hybrid Systems, vol. 3, no. 4, pp. 510-530, 2009.

[19] J.-W. Peng and J.-C. Yao, "A new hybrid-extragradient method for generalized mixed equilibrium problems, fixed point problems and variational inequality problems," Taiwanese Journal of Mathematics, vol. 12, no. 6, pp. 1401-1432, 2008.

[20] J.-W. Peng and J.-C. Yao, "Some new iterative algorithms for generalized mixed equilibrium problems with strict pseudo-contractions and monotone mappings," Taiwanese Journal of Mathematics, vol. 13, no. 5, pp. 1537-1582, 2009.

[21] Y. Yao, Y.-C. Liou, and J.-C. Yao, "A new hybrid iterative algorithm for fixed-point problems, variational inequality problems, and mixed equilibrium problems," Fixed Point Theory and Applications, vol. 2008, Article ID 417089, 15 pages, 2008.

[22] Y. Yao, Y.-C. Liou, and Y.-J. Wu, "An extragradient method for mixed equilibrium problems and fixed point problems," Fixed Point Theory and Applications, vol. 2009, Article ID 632819, 15 pages, 2009.

[23] W. Takahashi, Y. Takeuchi, and R. Kubota, "Strong convergence theorems by hybrid methods for families of nonexpansive mappings in Hilbert spaces," Journal of Mathematical Analysis and Applications, vol. 341, no. 1, pp. 276-286, 2008.

[24] S. Takahashi and W. Takahashi, "Strong convergence theorem for a generalized equilibrium problem and a nonexpansive mapping in a Hilbert space," Nonlinear Analysis: Theory, Methods E Applications, vol. 69 , no. 3, pp. 1025-1033, 2008.

[25] S.-S. Zhang, J. H. W. Lee, and C. K. Chan, "Algorithms of common solutions to quasi variational inclusion and fixed point problems," Applied Mathematics and Mechanics. English Edition, vol. 29, no. 5, pp. 571-581, 2008.

[26] J.-W. Peng, Y. Wang, D. S. Shyu, and J.-C. Yao, “Common solutions of an iterative scheme for variational inclusions, equilibrium problems, and fixed point problems," Journal of Inequalities and Applications, vol. 2008, Article ID 720371, 15 pages, 2008.

[27] P. Kumam and P. Katchang, "A general iterative method of fixed points for mixed equilibrium problems and variational inclusion problems," Journal of Inequalities and Applications, vol. 2010, Article ID 370197, 25 pages, 2010.

[28] G. L. Acedo and H.-K. Xu, "Iterative methods for strict pseudo-contractions in Hilbert spaces," Nonlinear Analysis: Theory, Methods \& Applications, vol. 67, no. 7, pp. 2258-2271, 2007.

[29] Z. Opial, "Weak convergence of the sequence of successive approximations for nonexpansive mappings," Bulletin of the American Mathematical Society, vol. 73, pp. 591-597, 1967.

[30] W. Takahashi, Nonlinear Functional Analysis, Yokohama Publishers, Yokohama, Japan, 2000.

[31] J.-W. Peng, Y. C. Liou, and J. C. Yao, "An iterative algorithm combining viscosity method with parallel method for a generalized equilibrium problem and strict pseudocontractions," Fixed Point Theory and Applications, vol. 2009, Article ID 794178, 21 pages, 2009.

[32] S. Atsushiba and W. Takahashi, "Strong convergence theorems for a finite family of nonexpansive mappings and applications," Indian Journal of Mathematics, vol. 41, no. 3, pp. 435-453, 1999. 